\title{
A VANISHING THEOREM
}

\author{
F. LAYTIMI AND W. NAHM
}

\begin{abstract}
The main result is a general vanishing theorem for the Dolbeault cohomology of an ample vector bundle obtained as a tensor product of exterior powers of some vector bundles. It is also shown that the conditions for the vanishing given by this theorem are optimal for some parameter values.
\end{abstract}

\section{$\S 1$. Introduction}

The main result of the paper is a vanishing theorem, which is the strongest possible immediate generalization of the vanishing theorems of Sommese [13], Manivel [11], Le Potier [8] and Laytimi-Nahm [7].

Let $E_{i}$ be vector bundles of ranks $d_{i}, i=1, \ldots, m$ over a compact complex manifold $X$ of dimension $n$ and $L$ a line bundle on $X$.

Theorem 1.1. If $\bigotimes_{i=1}^{m} \bigwedge^{r_{i}} E_{i} \otimes L$ is ample, then

$$
H^{p, q}\left(X, \bigotimes_{i=1}^{m} \wedge^{r_{i}} E_{i} \otimes L\right)=0 \quad \text { for } \quad p+q-n>\sum_{i=1}^{m} r_{i}\left(d_{i}-r_{i}\right) .
$$

One might suppress the explicit mention of the line bundle $L$ by including it among the $E_{i}$ as a vector bundle of rank 1 .

Sommese's result yields the vanishing with the same inequality for $p+q$ but with all $E_{i}$ ample. The ampleness of $E_{i}$ is not equivalent to that of $\bigwedge^{r_{i}} E_{i}$; for example the universal rank-r bundle $Q$ on the Grassmannian $G(r, d)$ is not ample, but $\Lambda^{r} Q$ is. Moreover, as follows from our theorem, it suffices to assume only that the tensor product of several terms of the form $\bigwedge^{r_{i}} E_{i}$ is ample.

The result of Le Potier is a particular case of Sommese's theorem for $m=1$.

For $p=n$ and all $E_{i}, i=1,2, \ldots, m$ ample vector bundles, EinLazarsfeld obtained less restrictive vanishing condition on $q$ [4].

Received September 21, 2004.

2000 Mathematics Subject Classification: 14F17. 
With our ampleness condition we conjecture that Theorem 1.1 is the best possible, as explained in Section 4 .

A partition $R=\left(r_{1}, r_{2}, \ldots, r_{m}\right)$ is a sequence of decreasing positive integers $r_{i}$, its length is $m$ and its weight is $|R|=\sum_{i=1}^{m} r_{i}$. Let $\tilde{R}$ be the transposed partition.

To each partition $R$ corresponds the canonical irreducible $G l(V)$-module $S_{R}(V)$. The functor $S_{R}$ is called the Schur functor (for a precise definition see [5, p. 45]). In particular $S^{k} V=\mathcal{S}_{(k)} V$, and $\bigwedge^{h} V=\underbrace{}_{\mathcal{S}_{(\text {times }}^{(1,1, \ldots, 1)}} V$.

We use the notation $\bigwedge_{R}=S_{\tilde{R}}$, where $\tilde{R}$ is the transpose of the partition $R$.

Schur functors were initially defined on the category of vector spaces and linear maps, but by functoriality the definition carries over to vector bundles on $X$.

THEOREM 1.2. Let $E$ be a vector bundle of rank $d$ over a compact complex manifold $X$ of dimension $n$ and $L$ a line bundle on $X$, then for any partition $\tilde{R}=\left(r_{1}, r_{2}, \ldots, r_{m}\right)$, if $\bigwedge_{R} E \otimes L$ is ample, then

$$
H^{p, q}\left(X, \bigwedge_{R} E \otimes L\right)=0 \quad \text { for } \quad p+q-n>\sum_{i=1}^{m} r_{i}\left(d-r_{i}\right) .
$$

This is Manivel's vanishing theorem with a weakened hypothesis; we do not assume the ampleness of $E$, as Manivel does. In fact Manivel's result is an immediate consequence of Sommese's.

It is crucial to have the optimal ampleness hypothesis as well as the optimal vanishing conditions in a vanishing theorem, specially for the geometrical applications like the study of degeneracy loci [6].

\section{§2. Proof of Theorem 1.1}

Let $E_{i}$ be as above and $Y_{i}=G r_{i}\left(E_{i}\right)$ the relative Grassmannian of subspaces of codimension $r_{i}$ in the fibers of $E_{i}$. Let $Q_{i}$ be the universal quotient bundles over $Y_{i}$.

LEMMA 2.1. Let $L$ be a line bundle on $X$,

$$
\begin{aligned}
& \pi_{i}: G r_{1}\left(E_{1}\right) \times_{X} \cdots \times_{X} G r_{m}\left(E_{m}\right) \longrightarrow G r_{i}\left(E_{i}\right), \\
& \pi: G r_{1}\left(E_{1}\right) \times_{X} \cdots \times_{X} G r_{m}\left(E_{m}\right) \longrightarrow X
\end{aligned}
$$

the natural maps. Then

$$
\bigotimes_{i=1}^{m} \Lambda^{r_{i}} E_{i} \otimes L \text { ample } \Longrightarrow \bigotimes_{i=1}^{m} \pi_{i}^{*}\left(\operatorname{det} Q_{i}\right) \otimes \pi^{*} L \text { ample }
$$


Proof. Let us introduce the following notations

$$
\begin{aligned}
W & =\bigotimes_{i=1}^{m} \Lambda^{r_{i}} E_{i} \otimes L \\
Y & =G r_{1}\left(E_{1}\right) \times_{X} \cdots \times_{X} G r_{m}\left(E_{m}\right) \\
Z & =\mathbb{P}\left(\Lambda^{r_{1}} E_{1}\right) \times \cdots \times \mathbb{P}\left(\Lambda^{r_{m}} E_{m}\right) \\
T & =\mathbb{P}\left(\Lambda^{r_{1}} E_{1} \otimes \cdots \otimes \Lambda^{r_{m}} E_{m}\right) \\
Y_{i} & =G r_{i}\left(E_{i}\right) \\
Z_{i} & =\mathbb{P}\left(\Lambda^{r_{i}} E_{i}\right) \\
\phi & : Y \longrightarrow Z \text { the Plücker embedding } \\
\gamma & : Z \longrightarrow T \text { the Segre embedding. }
\end{aligned}
$$

The commutative diagram

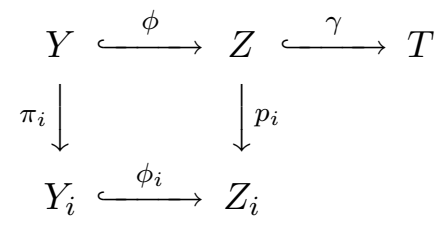

yields

$$
\begin{aligned}
\left.\mathcal{O}_{T}(1)\right|_{Y} & \simeq \phi^{*}\left(\bigotimes_{i=1}^{m} p_{i}^{*}\left(\mathcal{O}_{Z_{i}}(1)\right)\right) \\
& \simeq \bigotimes_{i=1}^{m} \pi_{i}^{*}\left(\phi_{i}^{*} \mathcal{O}_{Z_{i}}(1)\right) \simeq \bigotimes_{i=1}^{m} \pi_{i}^{*}\left(\operatorname{det} Q_{i}\right)
\end{aligned}
$$

Hence

$$
\left.\left.\mathcal{O}_{\mathbb{P}(W)}(1)\right|_{Y} \simeq \mathcal{O}_{T}(1)\right|_{Y} \otimes \pi^{*} L \simeq \bigotimes_{i=1}^{m} \pi_{i}^{*}\left(\operatorname{det} Q_{i}\right) \otimes \pi^{*} L
$$

and we are done.

For the sequel we need to recall the Borel-Le Potier spectral sequence in our context.

The projection $\pi: Y \rightarrow X$ yields a filtration of the bundle $\Omega_{Y}^{P}$ of exterior differential forms of degree $P$ on $Y$, namely

$$
F^{p}\left(\Omega_{Y}^{P}\right)=\pi^{*} \Omega_{X}^{p} \wedge \Omega_{Y}^{P-p}
$$

The corresponding graded bundle is given by

$$
F^{p}\left(\Omega_{Y}^{P}\right) / F^{p+1}\left(\Omega_{Y}^{P}\right)=\pi^{*} \Omega_{X}^{p} \otimes \Omega_{Y / X}^{P-p},
$$


$\Omega_{Y / X}^{P-p}$ is the bundle of relative differential forms of degree $P-p$. For a given line bundle $\mathcal{L}$ over $Y$, the filtration on $\Omega_{Y}^{P}$ induces a filtration on $\Omega_{Y}^{P} \otimes \mathcal{L}$. This latter filtration yields the Borel-Le Potier spectral sequence, which abuts to $H^{P, q}(Y, \mathcal{L})$. It is given by the data $X, Y, \mathcal{L}, P$ and will be denoted by $P \mathcal{E}_{B}$. Its $\mathcal{E}_{1}$-terms

$$
{ }^{P} \mathcal{E}_{1, B}^{p, q-p}=H^{q}\left(Y, \pi^{*}\left(\Omega_{X}^{p}\right) \otimes \Omega_{Y / X}^{P-p} \otimes \mathcal{L}\right)
$$

can be calculated as limit groups of the Leray spectral sequence ${ }^{p, P} \mathcal{E}_{L}$ associated to the projection $\pi$, for which

$$
{ }^{p, P} \mathcal{E}_{2, L}^{q-j, j}=H^{p, q-j}\left(X, R^{j} \pi_{*}\left(\Omega_{Y / X}^{P-p} \otimes \mathcal{L}\right)\right) .
$$

Now we start to prove the main theorem.

Denote the fibers of $E_{i}$ at a point $x \in X$ by $V_{i}$, and consider the line bundle on $Y \mathcal{L}=\bigotimes_{i=1}^{m} \pi_{i}^{*}\left(\operatorname{det} Q_{i}\right) \otimes \pi^{*} L$. We have

$$
\begin{aligned}
& R^{j} \pi_{*}\left(\Omega_{Y / X}^{P-p} \otimes \mathcal{L}\right) \\
& \quad \simeq H^{j}\left(G r_{1}\left(V_{1}\right) \times \cdots \times G r_{m}\left(V_{m}\right), \Omega^{P-p} \bigotimes_{i=1}^{m} \operatorname{det} Q_{i}\right) \otimes L .
\end{aligned}
$$

Using

$$
H^{p, q}(G r(V), \operatorname{det} Q)=0 \quad \text { if } \quad(p, q) \neq(0,0),
$$

and the Künneth formula, we get the degeneracy at the first step of both Leray and Borel spectral sequences. Now

$$
H^{0}\left(G r_{i}\left(V_{i}\right), \operatorname{det} Q_{i}\right) \simeq \bigwedge^{r_{i}} V_{i}
$$

thus

$$
H^{p, q}(Y, \mathcal{L}) \simeq H^{p, q}\left(X, \bigotimes_{i=1}^{m} \bigwedge^{r_{i}} E_{i} \otimes L\right)
$$

The result follows from Lemma 2.1 and Kodaira-Akizuki-Nakano vanishing theorem [1].

\section{§3. Proof of the Theorem 1.2}

Let's state this particular case of Theorem 1.1 as a

Corollary 3.1. Let $E$ be vector bundles of ranks $d$ over a compact complex manifold $X$ of dimension $n$ and $L$ a line bundle on $X$, then if $\bigotimes_{i=1}^{m} \bigwedge^{r_{i}} E \otimes L$ is ample, then

$$
H^{p, q}\left(X, \bigotimes_{i=1}^{m} \bigwedge^{r_{i}} E \otimes L\right)=0 \quad \text { for } \quad p+q-n>\sum_{i=1}^{m} r_{i}\left(d-r_{i}\right) .
$$


Actually we prove below

$$
\text { Corollary } 3.1 \Longleftrightarrow \text { Theorem } 1.2
$$

Recall the definition of the dominance partial order for arbitrary partitions [7].

Definition 3.2. Let $I=\left(i_{1}, i_{2}, \ldots\right), J=\left(j_{1}, j_{2}, \ldots\right)$ be any partitions of arbitrary weights. We define the dominance relation

$$
I \preceq J \quad \text { if for all } l \frac{i_{1}+i_{2}+\cdots+i_{l}}{|I|} \leq \frac{j_{1}+j_{2}+\cdots+i_{l}}{|J|} .
$$

We write

$$
I \sim J \quad \text { if } \quad I \succeq J \text { and } I \preceq J .
$$

Modulo this equivalence, this dominance relation is a partial order.

We recall from [9] that

$$
\text { If }|I|=|J|, \quad \text { then } \quad(I \succeq J \Longleftrightarrow \tilde{I} \preceq \tilde{J}) \text {. }
$$

We have [7]:

TheOREM 3.3. Let $I, J$ be any partitions and $E$ a vector bundle.

If $I \succeq J$, then $\mathcal{S}_{I} E$ ample (resp. nef) $\Longrightarrow \mathcal{S}_{J} E$ ample (resp. nef).

In particular, if $I \sim J$, then

$$
\mathcal{S}_{I} E \text { ample (resp. nef) } \Longleftrightarrow \mathcal{S}_{J} E \text { ample (resp. nef). }
$$

This theorem gives in particular

$$
\mathcal{S}^{k} E \text { ample (resp. nef) } \Longleftrightarrow E \text { ample (resp. nef), }
$$

and for any $k \geq 0$

$$
\bigwedge^{m} E \text { ample (resp. nef) } \Longrightarrow \bigwedge^{m+k} E \text { ample (resp. nef). }
$$

LemMA 3.4. Let $\tilde{R}=\left(r_{1}, \ldots, r_{m}\right)$ be a partition, then

$$
\bigotimes_{i=1}^{m} \bigwedge^{r_{i}} E \text { ample (resp. nef) } \Longleftrightarrow \bigwedge_{R} E \text { ample (resp. nef). }
$$


Proof. For the direct implication, the vector bundle $\bigwedge_{R} E$ is a direct summand of $\bigotimes_{i=1}^{m} \wedge^{r_{i}} E$, by the Littlewood-Richardson rules. For the opposite direction, all direct summands $S_{\lambda} E$ appearing in $\bigotimes_{i=1}^{m} \bigwedge^{r_{i}} E$ satisfy by also Littlewood-Richardson rules $\lambda \preceq \tilde{R}$. Hence the result follows from Theorem 3.3.

Lemma 3.4 and the fact that the vector bundle $\bigwedge_{R} E$ is a direct summand of $\bigotimes_{i=1}^{m} \bigwedge^{r_{i}} E$, yields Theorem 1.2.

Conversely it is easy to see that Theorem 1.2 implies the special case of Theorem 1.1 for which $E_{i}=E, i=1, \ldots, m$. Indeed for any $\bigwedge_{\mu} E$ which is a direct summand of $\bigotimes_{i=1}^{m} \bigwedge^{r_{i}} E$, we have

$$
\mu \succeq R
$$

where the partitions $R=\left(r_{1}, r_{2}, \ldots, r_{m}\right), \mu=\left(\mu_{1}, \mu_{2}, \ldots, \mu_{l}\right), l \leq m$ have the same weight.

To get the wanted vanishing condition, we need to show that

$$
\sum_{i=1}^{m} r_{i}\left(d-r_{i}\right) \geq \sum_{i=1}^{m} \mu_{i}\left(d-\mu_{i}\right), \quad \text { with } \mu_{i}=0 \text { if } i>l
$$

or equivalently

$$
\sum_{i=1}^{m} \mu_{i}^{2} \geq \sum_{i=1}^{m} r_{i}^{2}
$$

When we write

$$
\mu_{i}=r_{i}+\left(\alpha_{i}-\alpha_{i-1}\right)
$$

with $\alpha_{0}=0$, the inequality $(* *)$ implies that the $\alpha_{i}$ are non-negative. With

$$
\sum_{i=1}^{m} \mu_{i}^{2}=\sum_{i=1}^{m} r_{i}^{2}+\sum_{i=1}^{m}\left(2 \alpha_{i}\left(r_{i}-r_{i+1}\right)+\left(\alpha_{i}-\alpha_{i-1}\right)^{2}\right)
$$

we are done.

Remark 3.5. This gives a very short proof of our result in [7]. It still uses Theorem 3.2 proved in [7].

The following examples show that the numerical condition obtained in Theorem 1.1 is optimal for certain triples $(p, q, n)$. 


\section{$\S 4$. Example}

Let $V$ be a complex vector space, $G_{d}(V)$ is the Grassmannian of codimension- $d$ subspaces of $V, Q$ and $S$ the universal quotient bundle and the universal subbundle on $G_{d}(V)$.

For the sequel we need to recall Proposition 3 in [10] which is a generalization of a method developed in [12].

Proposition 4.1. Let $r, d, l$ be integers such that $0 \leq r<d$, and

$$
P(d, l, r)=(l-1) \frac{d(d+1)}{2}-d l+l+r d-\left(\begin{array}{c}
r+1 \\
2
\end{array}\right) .
$$

Then if $p \geq P(d, l, r)$,

$$
H^{p, q}\left(G_{d}(V), \bigwedge^{r} Q \otimes(\operatorname{det} Q)^{l}\right)=\bigoplus_{\alpha=0}^{l-1} \bigoplus_{\varepsilon \in\{0,1\}_{r}^{d}} \delta_{p, p(d, l, \varepsilon, \alpha)} \delta_{q, q(d, l, \varepsilon, \alpha)} \mathcal{S}_{\beta} V
$$

Here $\{0,1\}_{r}^{d}$ is the set of sequences $\varepsilon=\left(\varepsilon_{1}, \ldots, \varepsilon_{d}\right), \varepsilon_{i} \in\{0,1\}$ with $\sum_{i=1}^{d} \varepsilon_{i}=r$,

$$
\begin{gathered}
p(d, l, \varepsilon, \alpha)=\sum_{i=1}^{d}(d+1-i) \varepsilon_{i}-d \alpha+(l-1) \frac{d(d+1)}{2}, \\
q(d, l, \varepsilon, \alpha)=p(d, l, \varepsilon, \alpha)-d(l-1)-r+\alpha, \quad \text { and } \\
\beta=(\alpha+1,1, \ldots, 1), \quad|\beta|=d l+r .
\end{gathered}
$$

Note that here we have removed the first condition of the Proposition 3 in [10] which is not necessary.

Let $a=(\underbrace{1, \ldots, 1}_{r \text { times }}, \underbrace{0, \ldots, 0}_{d-r \text { times }})$. We have $p(d, l, \varepsilon, \alpha) \leq p(d, l, a, 0)$, with equality only for $\varepsilon=a, \alpha=0$. The latter case gives the following

EXAMPLE 4.2. Let $V$ be a vector space of dimension $r+d$, with $r \leq d$. If $p=d r-\left(\begin{array}{c}r \\ 2\end{array}\right)$, and $q=p-r$, then

$$
p+q-\operatorname{dim} G_{d}(V)=r(d-r)
$$

and

$$
H^{p, q}\left(G_{d}(V), \bigwedge^{r} Q \otimes \operatorname{det} Q\right)=\operatorname{det} V .
$$

Moreover $\operatorname{det} Q \otimes \bigwedge^{r} Q$ is ample. 
A generalization of this example to products of Grassmannians by the use of the Künneth formula gives

EXAMPLE 4.3. Let $G=\times_{i=1}^{m} G_{d_{i}}\left(V_{i}\right)$, where $V_{i}$ are vector spaces of dimension $r_{i}+d_{i}$. Let $p_{0}=\sum_{i=1}^{m} p_{i}, q_{0}=\sum_{i=1}^{m} q_{i}$, where $p_{i}=r_{i} d_{i}-\left(\begin{array}{c}r_{i} \\ 2\end{array}\right)$ and $q_{i}=r_{i} d_{i}-\left(\begin{array}{c}r_{i}+1 \\ 2\end{array}\right)$. Then

$$
H^{p_{0}, q_{0}}\left(G, \bigotimes_{i=1}^{m}\left(\bigwedge^{r_{i}} Q_{i} \otimes \operatorname{det} Q_{i}\right)\right)=\bigotimes_{i=1}^{m} \operatorname{det} V_{i}
$$

and $p_{0}+q_{0}-\operatorname{dim} G=\sum_{i=1}^{m} r_{i}\left(d_{i}-r_{i}\right)$.

In the formula we have suppressed the symbol for the obvious pull back of the universal quotient bundles $Q_{i}$ to the product $G$.

It is well known that the Kodaira-Akizuki-Nakano vanishing theorem is optimal. In particular, for any triple $p^{\prime}, q^{\prime}, n^{\prime}$ with $p^{\prime}+q^{\prime}-n^{\prime} \leq 0$ one can find a Cartesian product of $n^{\prime}$ curves $X$ and an ample line bundle $L$ on $X$ such that $H^{p^{\prime}, q^{\prime}}(X, L) \neq 0$. Taking the Cartesian product of $X$ with the Grassmannian product $G$ of the previous example, one finds examples with

$$
H^{p, q}\left(X \times G, L \bigotimes_{i=1}^{m}\left(\bigwedge^{r_{i}} Q_{i} \otimes \operatorname{det} Q_{i}\right)\right) \neq 0
$$

for any triple $(p, q, n)$ such that $p \geq q_{0}, q \geq q_{0}$ and $p+q-n \leq \sum_{i=1}^{m} r_{i}\left(d_{i}-\right.$ $\left.r_{i}\right)$, with $p_{0}, q_{0}$ as above and $n=\operatorname{dim} X \times G$.

Finally one has for $\operatorname{dim} V=d+1$,

$$
H^{d, d-1}\left(G_{d} V, \Lambda^{d-1} Q^{*} \otimes(\operatorname{det} Q)^{2}\right)=\operatorname{det} V
$$

since $\Lambda^{d-1} Q^{*} \otimes \operatorname{det} Q=Q$. Note that this example is legitimate, since we only demand that $\Lambda^{d-1} Q^{*} \otimes(\operatorname{det} Q)^{2}$ is ample, without any assumption on $Q^{*}$.

These examples are sufficiently diverse such that we conjecture that Theorem 1.1 is optimal in the full non-trivial parameter range of the triples $(p, q, n)$.

\section{REFERENCES}

[1] Y. Akizuki and S. Nakano, Note on Kodaira-Spencer's proof of Lefschetz theorems, Proc. Jap. Acad., 30 (1954), 266-272.

[2] R. Bott, Homogeneous vector bundles, Ann. Math., 66 (1957), 203-248.

[3] B. Demazure, A very simple proof of Bott's theorem, Invent. Math., 33 (1976), 271-220. 
[4] L. Ein and R. Lazarsfeld, Syzygies and Koszul cohomology of smooth projective varieties of arbitrary dimension, Invent. Math., 111 (1993), 51-67.

[5] W. Fulton and J. Harris, Representation theory, a first course, Graduate texts in Mathematics, Springer Verlag, 1991.

[6] F. Laytimi, On degeneracy loci, International Journal of Mathematics, 6 vol. 7 (1998), 203-220.

[7] F. Laytimi and W. Nahm, A generalization of Le Potier's vanishing theorem, Manuscripta math., 113 (2004), 165-189.

[8] Le Potier, Cohomologie de la Grassmannienne à valeurs dans les puissances extérieures et symetriques du fibré universel, Math. Ann., 226 (1977), 257-270.

[9] I. G. Macdonald, Symmetrics Functions and Hall polynomials, Claredon Press, Oxford, 1976.

[10] L. Manivel, Un théorème d'annulation pour les puissances extérieures d'un fibré ample, J. reine angew. Math., 422 (1991), 91-116.

[11] L. Manivel, théorèmes d'annulation pour les fibrés associés à un fibré ample, Scuola superiore Pisa (1992), 515-565.

[12] D. Snow, Cohomology of twisted holomorphic forms on Grassmann manifolds and quadric hypersurfaces, Math. Ann., 276 (1986), 159-176.

[13] A. J. Sommese, Submanifold of Abelian Varieties, Math. Ann., 233 (1978), 229-256.

F. Laytimi

Mathématiques - bât. M2

Université Lille 1

F-59655 Villeneuve d'Ascq Cedex

France

laytimi@agat.univ-lille1.fr

W. Nahm

Dublin Institute for Advanced Studies

10 Burlington Road

Dublin 4

Ireland

wnahm@stp.dias. ie 